\title{
Molecular patterns of avian influenza A viruses
}

\author{
KOU Zheng ${ }^{1,2}$, LEI FuMin ${ }^{3}$, WANG ShengYue ${ }^{4}$, ZHOU YanHong ${ }^{1 \dagger} \&$ LI TianXian $^{2 \dagger}$ \\ ${ }^{1}$ Hubei Bioinformatics and Molecular Imaging Key Laboratory, Department of Systems Biology, Huazhong University of Science and \\ Technology, Wuhan 430074, China; \\ ${ }^{2}$ State Key Laboratory of Virology, Wuhan Institute of Virology, Chinese Academy of Sciences, Wuhan 430071, China; \\ ${ }^{3}$ Institute of Zoology, Chinese Academy of Sciences, Beijing 100080, China; \\ ${ }^{4}$ Chinese National Human Genome Center, Chinese Academy of Sciences, Shanghai 201203, China
}

Avian influenza A viruses could get across the species barrier and be fatal to humans. Highly pathogenic avian influenza H5N1 virus was an example. The mechanism of interspecies transmission is not clear as yet. In this research, the protein sequences of 237 influenza A viruses with different subtypes were transformed into pseudo-signals. The energy features were extracted by the method of wavelet packet decomposition and used for virus classification by the method of hierarchical clustering. The clustering results showed that five patterns existed in avian influenza A viruses, which associated with the phenotype of interspecies transmission, and that avian viruses with patterns $C$ and $E$ could across species barrier and those with patterns $A, B$ and $D$ might not have the abilities. The results could be used to construct an early warning system to predict the transmissibility of avian influenza $A$ viruses to humans.

avian influenza A virus, H5N1, wavelet packet decomposition, clustering, interspecies transmission

Influenza A virus contains eight segments of single-strand negative RNA. Segment 4 codes hemagglutinin (HA) gene and segment 6 codes neuraminidase (NA) gene. According to the antigenic characteristics of HA and NA, avian influenza $A$ virus has 16 subtypes HA and nine subtypes NA.

Highly pathogenic avian influenza $\mathrm{H} 5 \mathrm{~N} 1$ viruses appeared in Asia in $1996^{[1]}$ and spread to Europe and Africa recently. This leads to a large-scale culling of poultry to prevent further influenza outbreaks and serious economic loss to the poultry industry. It was believed before 1997 that avian influenza virus could not infect humans directly until the genes of avian influenza virus mixed with those of human viruses, which were deduced by swine, the intermediate host ${ }^{[2]}$. In 1997, the facts that avian influenza H5N1 viruses were isolated from infected human samples changed the viewpoint and proved that avian influenza $\mathrm{H} 5 \mathrm{~N} 1$ virus could across the species balk and infect human respiration systems fatally ${ }^{[3,4]}$. H5N1 viruses were continuously reported to cause human death in Asia from 2003, to which much social attention has been paid ${ }^{[5-8]}$. In April 2005, highly pathogenic avian influenza $\mathrm{H} 5 \mathrm{~N} 1$ virus broke out in wild bird populations in Qinghai Lake ${ }^{[9,10]}$, a major breeding site for migratory birds in western China. It was the first report that highly pathogenic avian influenza H5N1 viruses were isolated from the migratory birds. Moreover, the 1918 'Spanish' pandemic virus (H1N1 subtype) suggested that its inner proteins originated from avian ${ }^{[11]}$. Several similar mutations were observed in $\mathrm{H} 5 \mathrm{~N} 1$ viruses $^{[11]}$

Besides H5N1 viruses, avian influenza A viruses of other subtypes had been reported to infect humans directly. In 1999, low pathogenic avian influenza A H9N2 virus infection was confirmed in two children with in-

\footnotetext{
Received Noveember 22, 2007; accepted March 23, 2008 doi: 10.1007/s11434-008-0236-2

†Corresponding author (email: litx@wh.iov.cn, yhzhou@mail.hust.edu.cn) Supported by the Special Basic Research Funds of China (Grant No. 2007FY210700), Major Program of National Natural Science Foundation of China (Grant No. 90608020), the National Basic Research Program of China (Grant Nos. 2005CB523007 and 2005CB523004), Research Fund for the Doctoral Program of Higher Education of China (Grant No. 20050487037), the Knowledge Innovation Project of the Chinese Academy of Sciences (Grant No. KSCX2-YW-N-065) and the Sixth Framework Program in European (Grant Nos. SP5B-CT-2006-044161 and SP5B-CT-2006- 044405)
} 
fluenza-like illness ${ }^{[12]}$. In 2003, low pathogenic avian influenza A H9N2 virus infection was confirmed in a child in Hong Kong again ${ }^{[13]}$. In the same year, 89 people were reported to have H7N7 influenza virus infection in Netherlands ${ }^{[14]}$. The mechanism of inter specie transmission of avian influenza A viruses was still unclear. We need to understand how different subtypes and strains of avian influenza virus became capable of crossing host species barriers. Besides the biological experiments, computational methods might provide some useful information.

The wavelet packet method is a powerful tool for spectral analysis. And the generalization of wavelet decomposition offers a rich range of possibilities for signal analysis. Many applications of wavelet method have been found in the analysis of biology data ${ }^{[15]}$. Cluster algorithms, which were popular for the classification of biology data, had been used in the analysis of influenza sequences ${ }^{[16]}$. In this work, the protein sequences of influenza A viruses were transformed into pseudo-signals and the energy features were extracted by the method of wavelet packet decomposition and were used for virus classification by the method of hierarchical clustering (Figure 1). We wanted to see if some patterns, which were associated with the phenotype of interspecies transmission, existed among avian influenza A viruses with different subtypes.

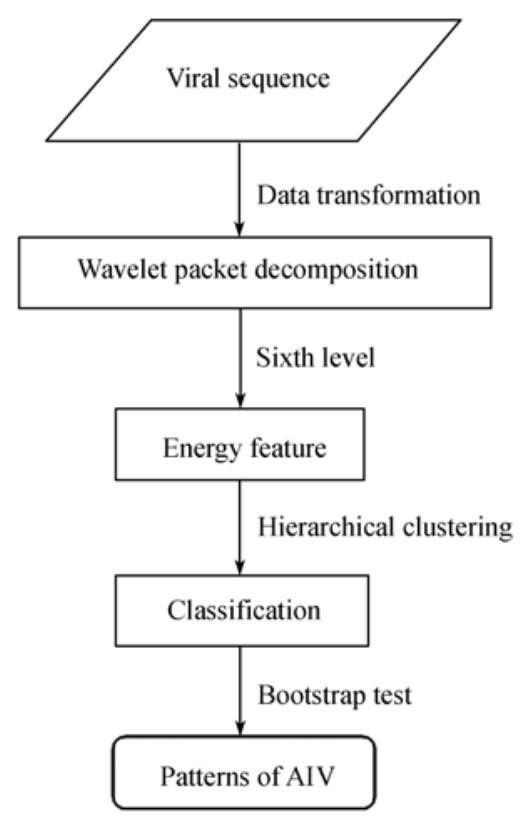

Figure 1 Flowchart of proposed methods

\section{Materials and methods}

\subsection{Data set}

One hundred and sixty H5N1 viruses isolated from 1996 to 2005 and 77 influenza A viruses of 5 other subtypes (H1N1, H2N2, H3N2, H7N7, and H9N2) isolated from 1918 to 2004 were used. Among the 237 viruses, 185 were isolated from avian, 34 from humans, 10 from swine, 4 from feline, and 4 from environment samples (Supplementary materials). The genome sequences of the 237 viruses were relatively full and covered the coding regions of six inner proteins (M1, NP, NS1, PA, PB1, PB2). All the nucleotide sequences were obtained from two public databases (FLAN, http://www.ncbi.nlm. nih.gov/genomes/FLU/FLU.html ${ }^{[17]}$; ISD, http://www.flu. lanl.gov $\left.{ }^{[18]}\right)$.

\subsection{Sequence transformation}

The nucleotide sequences of six inner proteins were aligned by using ClustalW 1.8 with default parameters. According to the ranges of the coding regions of the H5N1 virus (A/Goose/Guangdong/1/96), the extra sequences in the two terminals (upstream of start codon and downstream of end codon) were deleted. Then, the six inner protein sequences (M1, NP, NS1, PA, PB1, PB2) of each virus for further analysis were directly deduced from the nucleotide sequences according to the standard genetic code. Six protein sequences did not include gaps except NS1 protein (a 5-aa deletion). If several amino acid residues $(<5$ aa) were lacking in terminals, they were complemented by the conserved sequences, since viral proteins were conserved in the amidogen terminates and the carboxyl terminates.

The inner protein sequences of each virus were connected by the order: M1, NP, NS1, PA, PB1, PB2. Corresponding to the 237 viruses, 237 new viral sequences were generated respectively. The length of new sequence is 3212 (number of total lengths of six inner proteins). These biological sequences were transformed into numerical sequences with the same length according to the values of amino acid composition among the $160 \mathrm{H} 5 \mathrm{~N} 1$ viral protein sequences (Gap is replaced by zero; Table 1). Each numerical sequence was extended rightward with zero and the length became 4096 to adapt the wavelet packet analysis. 
Table 1 Amino acid composition among H5N1 viruses

\begin{tabular}{cccc}
\hline Amino acid & Composition(\%) & Amino acid & Composition(\%) \\
\hline Ala & 6.35 & Met & 4.48 \\
Cys & 1.32 & Asn & 4.92 \\
Asp & 4.57 & Pro & 3.95 \\
Glu & 7.82 & Gln & 4.04 \\
Phe & 3.94 & Arg & 7.55 \\
Gly & 6.26 & Ser & 7.00 \\
His & 1.45 & Thr & 6.53 \\
Ile & 6.23 & Val & 5.60 \\
Lys & 5.99 & Trp & 1.31 \\
Leu & 8.18 & Tyr & 2.53 \\
\hline
\end{tabular}

\subsection{Wavelet packet decomposition}

Numerical sequence for each virus was regarded as a pseudo signal. The signal was inputted into Matlab 7.0.1 for wavelet decomposition (the Details http://www. mathworks.com/products/wavelet/demos.html?file=/ products/demos/shipping/wavelet/waveletpacketsdemo. html). Daubechies 2 wavelet was used. The decomposition coefficients in the decomposition tree for each virus were got first. Then, the node energy in the decomposition tree was calculated by Gary's method ${ }^{[19]}$. Each influenza virus was respectively represented by the 64 node energy coefficients in the sixth level, which were used as sequence features for classification.

\subsection{Hierarchical Clustering}

The 237 node energy vectors were standardized before they were clustered. The Euclidean distance was assigned. Using their pair distances, we classified 237 avian influenza A viruses via an average-linkage clustering algorithm ${ }^{[20]}$. In this paper, a statistics package (pvclust) was used to assess the uncertainty in hierarchical cluster analysis ${ }^{[21]}$. For each cluster in the hierarchical clustering, $P$-values are calculated via 10000 multiscale bootstrap resamplings ${ }^{[22]}$. The $P$-value of a cluster is a value between 0 and 1, which indicates how strong the cluster is supported by data.

\section{Results}

\subsection{Classification of avian influenza A viruses}

With the method of wavelet packet decomposition, 237 viral sequences were transformed and were grouped into eight clusters with high bootstrap values $(>90 \%$, Figure 2).

As shown in Figure 2, cluster A was composed of three H5N1 viruses isolated from avian in 1996 and 2000, four H5N1 viruses isolated from environment in

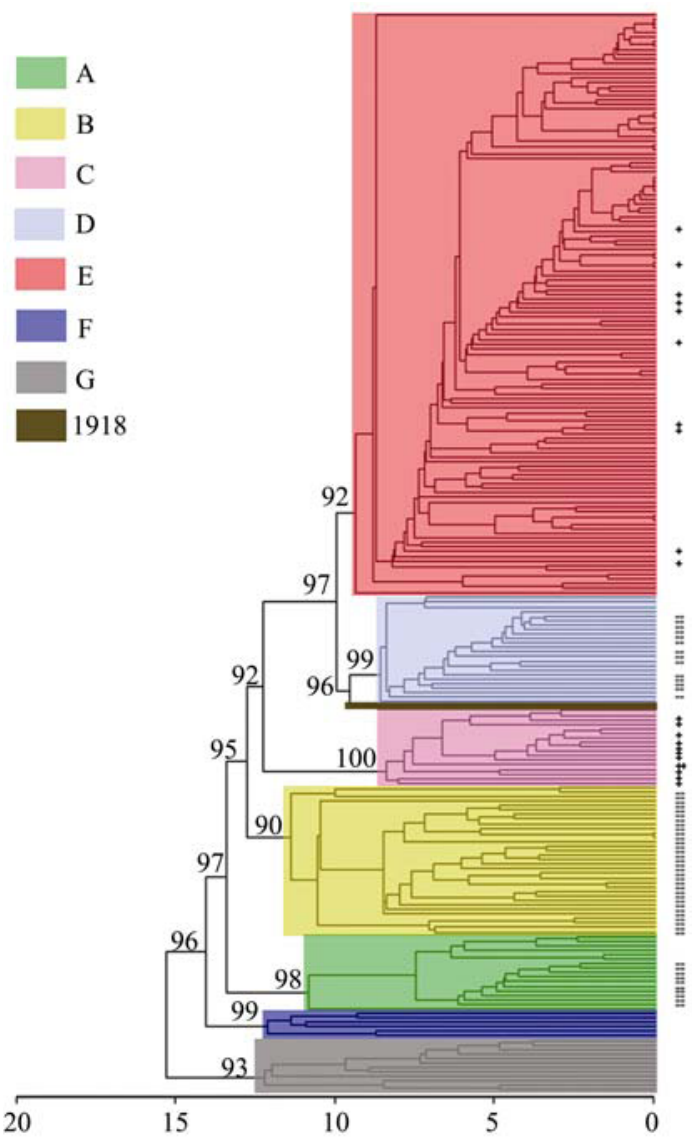

Figure 2 Hierarchical clustering of 237 influenza A viruses. The scale bar shows the average distance between clusters. Eight patterns are shown by eight colors. 20 human $\mathrm{H} 5 \mathrm{~N} 1$ viruses are marked by single plus. One human viruses, HK/1073/99 (H9N2), is marked by double plus. 56 avian viruses of five subtypes (H1N1, H2N2, H3N2, H7N7 and H9N2) are marked by double asterisks. The $P$-values (\%) of the main clusters based on 10000 bootstrap samplings are shown.

2000 and ten avian influenza viruses of three other subtypes (H1N1, H3N2, H9N2). Cluster B was composed of 32 avian influenza H9N2 viruses and one H5N1 virus isolated from swine in 2003. Ten H5N1 viruses isolated from human beings, six $\mathrm{H} 5 \mathrm{~N} 1$ viruses isolated from avian from 1997 to 1998 in Hong Kong, and one H9N2 virus (A/HK/1073/99(H9N2)) isolated from human beings made up cluster $\mathrm{C}$. Seven avian $\mathrm{H} 5 \mathrm{~N} 1$ viruses isolated from 2001 to 2004, 14 avian influenza viruses of four other subtypes (H1N1, H2N2, H7N7, H9N2), and one swine virus (A/swine/Korea/S452/2004(H9N2)) were included in cluster D. Ten H5N1 viruses isolated from humans and $111 \mathrm{H} 5 \mathrm{~N} 1$ viruses isolated from avian, swine and feline from 2000 were distributed into Cluster E. Six swine adaptive viruses and 12 human adaptive viruses separately formed cluster F and G. The 1918 'Spanish' virus formed a separate cluster close to cluster $\mathrm{D}$. 
The fact that avian influenza H5N1 and H9N2 viruses isolated from humans only appeared in cluster $\mathrm{C}$ and $\mathrm{E}$ while human or swine adaptive viruses formed separate clusters suggested that avian influenza $\mathrm{A}$ viruses in cluster $\mathrm{C}$ and $\mathrm{E}$ could directly infect human beings (Figure 2). The fact that 56 avian influenza viruses of five subtypes, which had not been reported to have the ability of infecting human, only distributed in cluster A, B and $D$ suggested that avian influenza $A$ viruses in cluster A, B, and D could not cross the species barrier (Figure 2). The 1918 'Spanish' virus formed a separate cluster and was close to avian influenza viruses, suggesting that the 1918 'Spanish' virus originated from avian directly ${ }^{[11]}$.

\subsection{Patterns of avian influenza A viruses}

The distributions of node energy coefficients were shown in Figure 3, where the red curves represent the mean values in each cluster. The energy distributions between eight clusters were different (Figure 3). Based on the results of clustering, it was supposed that five patterns (patterns $\mathrm{A}-\mathrm{E}$ ) existed among avian influenza A viruses and different from those of swine or human adaptive viruses (patterns $\mathrm{F}$ and $\mathrm{G}$ ).

Avian influenza $\mathrm{H} 5 \mathrm{~N} 1$ and $\mathrm{H} 9 \mathrm{~N} 2$ viruses isolated from humans only had pattern $\mathrm{C}$ or $\mathrm{E}$, suggesting that if one avian influenza A virus isolated in the future has an energy distribution similar to that of avian influenza viruses with pattern $\mathrm{C}$ or $\mathrm{E}$, it can directly infect humans. If one avian virus had an energy distribution similar to that of the '1918' Spanish pandemic virus, it can not only infect humans, but also is likely to cause a pandemic if hemagglutinin protein could bind to cellular receptors with 2,6-NeuAcGal linkages. However, avian influenza viruses with a distribution like that of avian influenza viruses with pattern $\mathrm{A}, \mathrm{B}$, or D could not infect humans. In short, five patterns, which were associated with the phenotype of interspecies transmission, existed among avian influenza A viruses. An early warning system to predict the transmissibility of avian influenza A viruses to humans could be constructed to assess whether one avian influenza virus had the possibility to across the species barrier.

\section{Discussion}

Most emerging infectious diseases in humans had animal reservoirs, such as highly pathogenic avian influenza H5N1 virus ${ }^{[23]}$. During the processes of becoming endemic in a new host species, three necessary stages could be involved: close contact between hosts of the donor and recipient species, host-virus interactions within individual hosts of the recipient species, and host-host transmission within the recipient species ${ }^{[24]}$. Some avian influenza A viruses could cross the barrier and stay in the second stage recently. To be ready for the next endemic or pandemic spread, we need to predict which subtypes and strains of avian influenza virus will become capable of crossing host species barriers.

In this paper, via wavelet packet decomposition and average-linkage clustering algorithm, the genome sequences of 237 influenza A viruses were transformed and classified in a novel way to explore patterns associated with the phenotype of interspecies transmission. The results of clustering suggested that five pattern existed in avian influenza A viruses with high bootstrap values (Figures 2 and 3). It was found that H5N1 and $\mathrm{H} 9 \mathrm{~N} 2$ viruses isolated from humans only had pattern $\mathrm{C}$ or $\mathrm{E}$ while human or swine adaptive viruses had pattern F, G or '1918' (Figure 2), and that 56 avian influenza viruses of five subtypes without ability of infecting humans had pattern A, B or D (Figure 2). We concluded that avian influenza $\mathrm{A}$ viruses with pattern $\mathrm{C}$ or $\mathrm{E}$ could have the ability of interspecies transmission while those with pattern A, B or D could not.

It was believed that avian influenza viruses achieved the ability of infecting humans by mutations although what mutations or key sites that play the key roles is unclear $^{[24]}$. Influenza viruses evolve in a fast speed while mammal hosts evolve in a very low speed. To escape the barrier, influenza viruses could not evolve randomly and they must abide to some unclear restrictions to adapt hosts. So, we hypothesize that different patterns exist among avian influenza viruses, belonging to two categories: one can infect humans and the other could not. The analysis results confirmed our hypothesis.

Fifty-six avian viruses of five subtypes (H1N1, H2N2, H3N2, H7N7 and H9N2) isolated from 1966 to 2004 were classified into cluster A, B or D (Figure 2) and had pattern A, B or D (Figure 3), suggesting that avian influenza A viruses in nature have three reserved patterns. The trends of avian influenza A viruses to escape the species barrier and form the pattern C, E or ' 1918 ' existed during the evolution history. The reason for failing to cross the species barrier was complicated and associated with many factors such as the structure of viral proteins, host itself and living environments. 

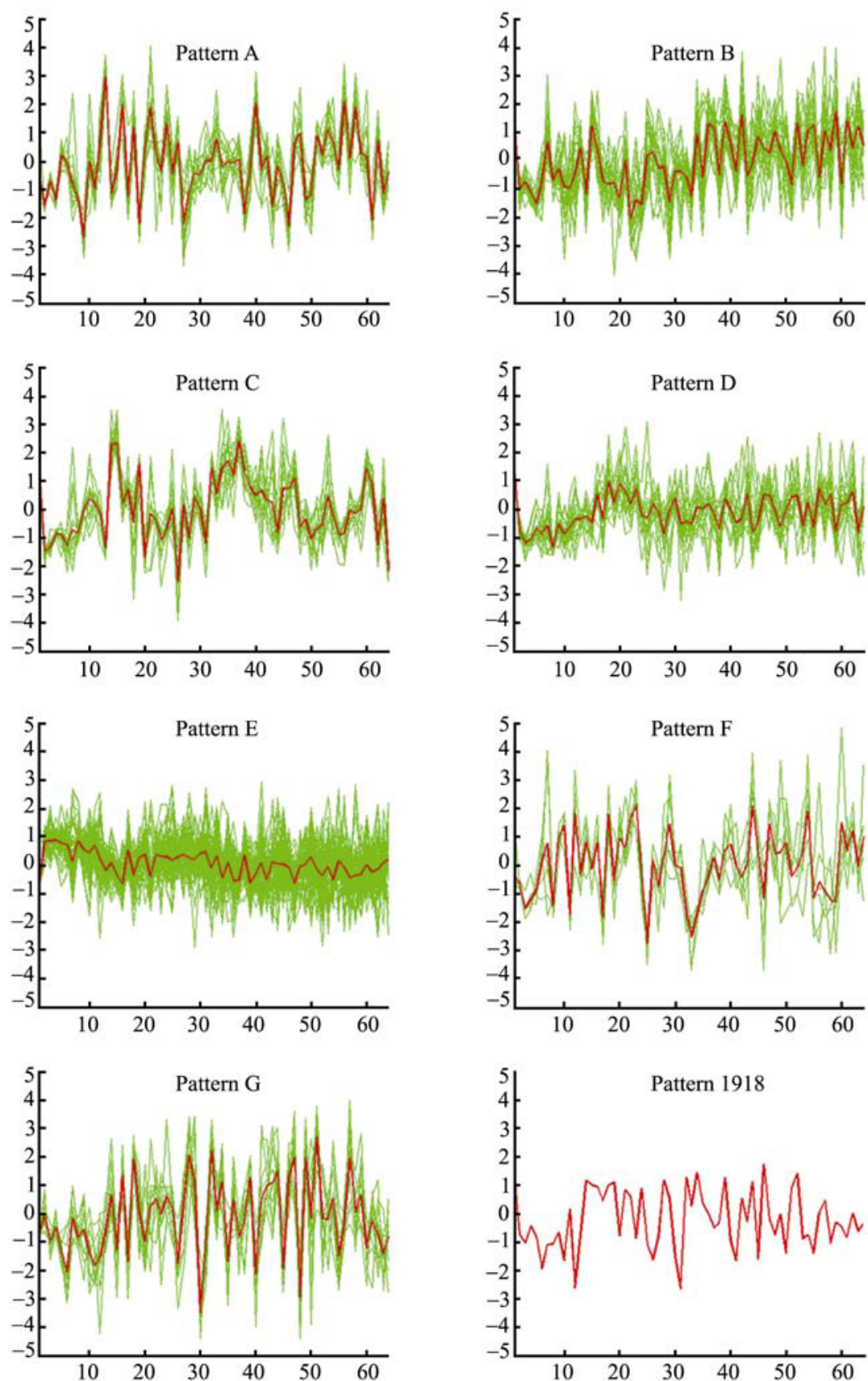

Figure 3 Patterns of avian influenza A viruses. The standardized values of node energy coefficients for each virus in eight patterns are shown by green curves. The $X$-axis represents the 64 nodes in the sixth levels. The $Y$-axis represents the standardized node energy. The mean values for each pattern are shown by red curves.

H5N1 viruses are continually epidemic in Asian, Europe, and Africa. Six gene segments coding inner proteins continued to be reassortment while two of outer proteins keep the preference of binding to cellular receptors with 2,3-NeuAcGal linkages ${ }^{[5,6,25,26]}$. The inner proteins could play a key role in the direct interspecies transmission of avian influenza A viruses to humans and shed light on the mechanisms. So, we only used the six inner protein sequences to classify avian influenza viruses.

The possibility of virus adaptating to recipient species was not simply determined by the overall rate at which mutations arise, but by the fitness or proportion of these mutations in key sites ${ }^{[24]}$. The traditional phylogenetic analysis was based on the mutation numbers in the genome or gene sequences, which were classical and useful for evolution analysis, but not for pattern recognition 
of the interspecies transmission of avian influenza A viruses $^{[6,24-26]}$. We previously analyzed the viral genomes by using Neighbor-Jointing clustering over a multiple genome sequence alignment with Kimura correction. The distributions of avian influenza A viruses were disordered and an explainable result is not available. We used the method described in the paper to classify avian influenza A viruses and got a relatively reasonable explanation. The reason might be that the features used for classification are not similar to those for the phylogenetic analysis.

In this work, we could not get some typical biological features from the method itself, although these features could result in a better understanding of how and why viruses jump the species barrier. The reason is that the wavelet packet decomposition transformed the biological sequences into an energy space and the relationship between biological features and energy values was weak.

1 Xu X, Subbarao K, Cox N J, et al. Genetic characterization of the pathogenic influenza A/Goose/Guangdong/1/96 (H5N1) virus: Similarity of its hemagglutinin gene to those of $\mathrm{H} 5 \mathrm{~N} 1$ viruses from the 1997 outbreaks in Hong Kong. Virology, 1999, 261: 15-19

2 Webster R G, Bean W J, Gorman O T, et al. Evolution and ecology of influenza A viruses. Microbiol Rev, 1992, 56: 152-179

3 Claas E C, Osterhaus A D, van Beek R, et al. Human influenza A $\mathrm{H} 5 \mathrm{~N} 1$ virus related to a highly pathogenic avian influenza virus. Lancet, 1998, 351: 472-477

4 Subbarao K, Klimov A, Katz J, et al. Characterization of an avian influenza A (H5N1) virus isolated from a child with a fatal respiratory illness. Science, 1998, 279: 393-396

5 Chen H, Smith G J, Li K S, et al. Establishment of multiple sublineages of $\mathrm{H} 5 \mathrm{~N} 1$ influenza virus in Asia: Implications for pandemic control. Proc Natl Acad Sci USA, 2006, 103: 2845-2850

6 Li K S, Guan Y, Wang J, et al. Genesis of a highly pathogenic and potentially pandemic H5N1 influenza virus in eastern Asia. Nature, 2004, 430: 209-213

7 Zhu Q Y, Qin E D, Wang W, et al. Fatal infection with influenza A (H5N1) virus in China. N Engl J Med, 2006, 354: 2731 - 2732

8 Shu Y L, Yu H J, Li D X. Lethal avian influenza A (H5N1) infection in a pregnant woman in anhui province, China. N Engl J Med, 2006, 354: $1421-1422$

9 Chen H, Smith G J, Zhang S Y, et al. Avian flu: H5N1 virus outbreak in migratory waterfowl. Nature, 2005, 436: 191-192

10 Liu J H, Xiao H, Lei F M, et al. Highly pathogenic H5N1 influenza virus infection in migratory birds. Science, 2005, 309: 1206

11 Taubenberger J K, Reid A H, Lourens R M, et al. Characterization of the 1918 influenza virus polymerase genes. Nature, 2005, 437: $889-893$

12 Lin Y P, Shaw M, Gregory V, et al. Avian-to-human transmission of H9N2 subtype influenza a viruses: Relationship between H9N2 and H5N1 human isolates. Proc Natl Acad Sci USA, 2000, 97: 9654-9658

13 Butt K M, Smith G J, Chen H, et al. Human Infection with an avian H9N2 influenza A virus in Hong Kong in 2003. J Clin Microbiol, 2005, 43: 5760-5767
This is the main limitation in the paper. Maybe, those sites with high similarity within individual patterns and high variation among patterns are the key sites for interspecies transmission ${ }^{[27]}$. We were not sure whether these sites in six inner proteins have clear or convinced relationship with the patterns or phenotypes about interspecies transmission until simulations based on mutations in silicon and biological tests are done.

Pattern in the paper is a quantitative description about the possibility of avian influenza A viruses crossing species barrier. Based on the analysis of the results, the category information about interspecies transmission of avian influenza A viruses was clear. A predicting system for early warning could be constructed based on various machine learning methods. The system could benefit public health.

We acknowledge Dr. HU Z H and Dr. Rayner S for helpful discussion.

14 Fouchier R A, Schneeberger P M, Rozendaal F W, et al. Avian influenza A virus (H7N7) associated with human conjunctivitis and a fatal case of acute respiratory distress syndrome. Proc Natl Acad Sci USA, 2004, 101: 1356-1361

15 Liờ $\mathrm{P}$. Wavelets in bioinformatics and computational biology: State of art and perspectives. Bioinformatics, 2003, 2003: 2-9

16 Plotkin J B, Dushoff J, Levin S A. Hemagglutinin sequence clusters and the antigenic evolution of influenza A virus. Proc Natl Acad Sci USA, 2002, 99: 6263-6268

17 Bao Y, Bolotov P, Dernovoy D, et al. FLAN: A web server for influenza virus genome annotation. Nucleic Acids Res, 2007, 35 W280-W284

18 Osterhaus A D M E, Cox N, Hampson A W. Options for the Control of Influenza IV. Amsterdam: Elsevier Science, 2001

19 Gary G Y, Kuo C L. Wavelet packet feature extraction for vibration monitoring. IEEE Transact Indust Electron, 2000, 47: 650-667

20 Sokal R R, Michener C D. A statistical method for evaluating systemic relationships. Univ Kansas Sci Bull, 1958, 38, 1409-1438

21 Suzuki R, Shimodaira H. Pvclust: An R package for assessing the uncertainty in hierarchical clustering. Bioinformatics, 2006, 22: $1540-1542$

22 Shimodaira H. Approximately unbiased tests of regions using multistep-multiscale bootstrap resampling. Annal Statist, 2004, 32: $2616-2641$

23 Kuiken T, Leighton F A, Fouchier R A, et al. Public health: Pathogen surveillance in animals. Science, 2005, 309: 1680-1681

24 Kuiken T, Holmes E C, McCauley J, et al. Host species barriers to influenza virus infections. Science, 2006, 312: 394-397

25 Guan Y, Peiris J S, Lipatov A S, et al. Emergence of multiple genotypes of H5N1 avian influenza viruses in Hong Kong SAR. Proc Natl Acad Sci USA, 2002, 99: 8950-8955

26 Kou Z, Lei F M, Yu J, et al. New Genotype of avian influenza H5N1 viruses isolated from tree sparrows in China. J Virol, 2005, 79: $15460-15466$

27 Finkelstein D B, Mukatira S, Mehta P K, et al. Persistent host markers in pandemic and H5N1 influenza viruses. J Virol, 2007, 81: $10292-10299$ 